\title{
An Internet-Based Self-Help Program for the Treatment of Fear of Public Speaking: A Case Study
}

\author{
C. Botella \\ M. J. Gallego \\ A. Garcia-Palacios \\ R. M. Baños \\ S. Quero \\ V. Guillen
}

\begin{abstract}
This article discusses the development of the first totally self-administered online CBT program for the treatment of a specific social phobia (fear of public speaking) called talk to me. The online program includes three parts. The assessment protocol gives the patient information about the problem, including impairment, severity,
\end{abstract}

C. Botella, Universitat Jaume I, Spain, Ciber Fisiopatologia Obesidad y Nutricion (CB06/03) Instituto Salud Carlos III, Spain.

M. J. Gallego, Universitat Jaume I, Spain, Ciber Fisiopatologia Obesidad y Nutricion (CB06/03) Instituto Salud Carlos III, Spain.

A. Garcia-Palacios, Universitat Jaume I, Spain, Ciber Fisiopatologia Obesidad y Nutricion (CB06/03) Instituto Salud Carlos III, Spain.

R. M. Baños, Universidad de Valencia, Spain, Ciber Fisiopatologia Obesidad y Nutricion (CB06/03) Instituto Salud Carlos III, Spain.

S. Quero, Universitat Jaume I, Spain, Ciber Fisiopatologia Obesidad y Nutricion (CB06/03) Instituto Salud Carlos III, Spain.

V. Guillen, Centro Clínico PREVI, Spain.

Address correspondence to Cristina Botella, Dpt. Psicologia Basica, Clinica y Psicobiologia, Universitat Jaume I. Avda Vicent Sos Baynat s/n. 12071 Castellon, Spain. E-mail: botella@psb.uji.es.

Journal of Technology in Human Services, Vol. 26(2/4) 2008

Available online at http://jths.haworthpress.com

(C) 2008 by The Haworth Press. All rights reserved. 
and the degree of fear and avoidance regarding the main feared situations. The structured treatment protocol ensures that the patient does not skip any steps in the treatment. The treatment protocol is a CBT program that provides exposure to the feared situation using videos of real audiences. Finally, the control protocol assesses treatment efficacy, not only at posttreatment, but also at every intermediate step. In this work we describe talk to Me and its practical application through a case study.

KEYWORDS. Cognitive-behavioral therapy, exposure, fear of public speaking, online therapy, social phobia, telepsychology

Social phobia is the third most prevalent mental disorder, after major depressive disorder and alcohol dependence (Kessler et al., 1994). An estimated 13.3 percent of the population in United States experiences social phobia at some point in their lives (Magee, Eaton, Wittchen, McGonagle, \& Kessler, 1996). The essential feature of social phobia is persistent and intense fear of one or more social situations in which the person is exposed to the observation of others (APA, 2000). DSM-IV-TR specified a generalized subtype of social phobia, which is diagnosed when someone fears all or almost all social situations. However, the variability of social situations feared by social phobics has opened up a discussion on the necessity of distinguishing among different social phobia subtypes. Heimberg, Holt, Scheneier, Spitzer, and Liebowitz (1993) distinguished three social phobia subtypes: generalized social phobia, which includes fear across almost all social situations; nongeneralized social phobia, which includes fear of multiple social situations, but no problems in at least one social domain; and finally, discrete (or specific) social phobia, which includes fear in only one or two circumscribed social situations (e.g., public speaking or eating in public). In this article we have centered our attention on fear of public speaking. This is the most feared situation among the general population. Its prevalence ranges from 20 percent (Cho \& Won, 1997; Pollard \& Henderson, 1988) to 34 percent (Stein, Walter, \& Forde, 1996).

Cognitive behavioural therapy (CBT) including exposure, specifically in vivo exposure, is considered the treatment of choice for 
social phobia and specific phobias. Regarding social phobia this intervention has received wide empirical support from numerous clinical trials (e.g., Butler, Cullington, Munby, Amies, \& Gelder, 1984; Mattick, Peters, \& Clarke, 1989; Turner, Beidel, \& Jacob, 1994). The APA report on empirically supported treatments (Task Force on Promotion and Dissemination of Psychological Procedures, 1995, last updated by Woody Barlow, 1997; Heimberg, 1991; Marks, 1978b; Turner, Beidel, \& Cooley, 1997).

In vivo exposure consists of confronting the feared situation in a gradual and systematic way. It begins with lower-ranked situations and moves up to more highly feared situations. In a typical exposure session the therapist encourages the patient to confront the feared situation. The therapist asks the patient about the degree of fear from 0 to 10 (or 0 to 100) using the subjective units of discomfort (SUDs; Wolpe, 1969) every few minutes. When fear goes down significantly the patient can move on to confront a more difficult situation. Exposure therapy is based on the notion that individuals are able to adjust to anxiety-provoking stimuli through a process known as habituation (Marks, 1987). Foa and Kozak (1986) used the concept of emotional processing to explain fear reduction during exposure. They support the hypothesis that exposure to feared stimuli allows the activation of the fear structure and the presentation of corrective information incompatible with the pathological elements of the fear structure.

In vivo exposure is an effective therapy technique, although it is not free of limitations. Some patients (approximately 25 percent of those who start an exposure program) refuse exposure therapy or drop out of therapy (Marks, 1978a, 1992). One reason for this percentage of refusal could be that the main feature of exposure is confronting the feared stimuli; some people may find this too frightening. Furthermore, the vast majority (approximately 60 to 85 percent) of those afflicted with specific or social phobias never seek treatment for their problem (Boyd et. al., 1990; Magee et al., 1996). In the case of social phobics, they may abstain from seeking treatment because of the embarrassment associated with meeting an unknown person, the psychologist. Finally, in vivo exposure programs and other CBT programs entail an important amount of therapy time. This means an important financial cost for patients and public mental health institutions. Also, it is difficult for some patients living in remote areas (i.e., rural areas) to get CBT treatment. An important goal in 
clinical psychology is reducing cost of treatment without decreasing effectiveness. The main factor to minimize economic issues is reducing contact with the therapist (Al-Kubaiy et al., 1992; Marks, 1987; Öst, Salkovskis, \& Hellström, 1991). The length of the therapist contact has varied from one visit per week to structure new exposure tasks (Mathews, Gelder, \& Johnston, 1981) to no contact at all during the treatment (Ghosh \& Marks, 1987; Hellström \& Üst, 1995; Öst et al., 1991). Self-directed exposure has shown to be as successful as standard therapist-directed treatment (Ghosh \& Marks, 1987). In another study the improvement achieved by self-directed exposure was maintained at two-year follow-up (Park et al., 2001).

One way of reducing therapy contact time and overcoming some of these limitations is telepsychology. Telepsychology has been defined as "the use of telecommunication technologies to put patients in contact with the mental health practitioners with the aim of providing a suitable diagnosis, education, treatment, consultations, communication and storage of the patients' records, research data, and other activities" (Brown, 1998, p. 963). One way to deliver telepsychology is though the Internet (online therapy). Recently, Schneider, Mataix-Cols, Marks, and Bachofen (2005) compared two Internetguided self-help treatments for phobic and panic disorders, one included exposure instructions and the other did not. They found that at posttest both were equally effective, however at one-month followup the Internet-guided self-help treatment with exposure instructions was more effective than the other.

In the field of social phobia there are some studies that reported data on the use of online telepsychology programs to treat this disorder. These studies could be classified in two groups according to the classification of Glasgow and Rosen (1978): (1) Internet-based self-help programs with therapist contact, and (2) Internet-based selfhelp programs without therapist contact.

Regarding the first group, Andersson et al. (2006) combined an Internet-based self-help program with therapist contact via e-mail with two group exposure sessions. This treatment showed its efficacy in a controlled randomized study. The same Internet program plus weekly therapist contact via e-mail without the group exposure sessions was administered to 26 social phobics (Carlbring, Furmark, Stezkó, Ekselius, \& Andersson, 2006). The participants improved significantly from pre- to posttest and the results were maintained at sixmonth follow-up. Carlbring et al. (2007) compared in a controlled 
randomized study their Internet program plus weekly phone calls with a waiting-list group. They also found this treatment effective to treat social phobia, and it improved program adherence. This improvement was maintained after one year.

There is only one telepsychology treatment program completely delivered over the Internet to treat social phobia (Botella et al., 2000). It is an Internet-based self-help program for the treatment of fear of public speaking called Talk to Me, designed by our team. This treatment has shown preliminary efficacy in a case study (Botella, Hofmann, \& Moscovitch, 2004), and two single case series (Gallego et al., 2007; Guillen, 2001). In these studies there are no data of within-exposure sessions.

The aim of the present study is to describe the Talk to Me program and its application to a person with social phobia, with the most important contribution being presenting data of within-exposure sessions in order to show how the program is able to activate anxiety and promote habituation.

\section{METHOD}

\section{Participant}

The patient is a 30 -year-old Caucasian woman. She is an undergraduate student. She lives with her husband in a Spanish town. An important issue in the history of the problem is that she did not need to confront a public speaking situation until she began her studies at the university. The problem appeared three years ago when she had to present a project in class. She remembers that it was a very bad experience because she was very nervous and noticed that her heart beat very fast, her hands sweat, and her face got red. At that moment the patient was concerned about her anxiety symptoms, and she thought that she was acting in a humiliating way. Furthermore, she found it difficult to center her attention on the project because thoughts such as "they are evaluating me negatively" or "I am doing it badly" were crossing her mind. After this situation the patient feared and avoided public speaking situations. The patient looked for help when she realized that the problem impaired her studies. At the time she was afraid of attending some classes because she knew that speaking in public was possible. In one class, students 
were assigned to perform a role-play simulating a therapist-patient interaction. The patient felt very nervous during these classes and she avoided performing the role-play. Another feared situation was when the professor asked questions in class; then she felt very nervous thinking of the possibility of having to speak.

In the initial assessment she met DSM-IV-TR (APA, 2000) criteria for social phobia. Two experienced clinicians performed two independent interviews using a structured interview (see the measures section), and both of them confirmed the diagnosis. Taking into account Heimberg and colleagues' (1993) classification, both therapists agreed on the diagnosis of discrete social phobia. The patient did not present comorbidity with any other diagnosis. Regarding the scores in some measures of fear of public speaking (selfstatements during public speaking [SSPS], personal report of confidence as a speaker [PRCS], and public speaking self-efficacy questionnaire [PSSEQ]; see measures in the following section), the patient's scores were similar to the scores found in clinical population suffering social phobia. Finally, patient and therapist selected two target behaviors. The first one was presenting a project in class and the second one was giving a talk to high school students or their parents. The main catastrophic thought associated to each of them was "People will think I'm stupid."

\section{Measures}

We used several instruments in order to have a thorough assessment of the patient's problem. In this section we describe those instruments. The key assessment instruments of Talk to Me are marked with an asterisk.

Consent Form. The participant read and signed a consent form.

*Diagnosis: Anxiety disorders interview schedule IV (ADIS-IV; Di Nardo, Brown, \& Barlow, 1994). We used an adapted version of this semistructured interview that assesses the DSM-IV-TR (APA, 2000) criteria for social phobia and the degree of severity of the problem.

Brief version of the fear of negative evaluation scale (BFNE) (Leary, 1983). This scale assesses apprehension at the prospect of being negatively evaluated by others. It is comprised of 12 items that are 
rated on a scale of $1-5$, where 1 is "not at all characteristic of me" and 5 is "extremely characteristic of me." This instrument has shown good psychometric properties in a clinical Spanish population (Botella \& Gallego, 2007).

Self-statements during public speaking (SSPS) (Hofmann \& DiBartolo, 2000). This scale is composed of 10 items that are rated in $1-5$ scales, where 1 is "I completely disagree" and 5 is "I completely agree." It measures self-statements and levels of discomfort while someone is speaking in public. This instrument is comprised of two subscales: positive self-statements subscale (SSPS-P) and negative self-statements subscale (SSPS-N). Both subscales have excellent internal consistency (SSPS-P $\alpha=.80$; SSPS-N $\alpha=.86$ ) and test-retest reliability (SSPS-P r $=.78$; SSPS-N r $=.80$ ) (Hofmann \& DiBartolo, 2000).

Personal report of confidence as a speaker (PRCS) (Paul, 1966). This measure was developed by Gilkinson (1942) as a 104-item self-report measure of fear of public speaking. Paul (1966) shortened this instrument to 30 true-false items. In Spain, Bados (1986) changed the true-false items format for a six-point scale (1 is "not at all characteristic of me," and 10 is "extremely characteristic of me"). This instrument is validated in Spanish populations by Méndez, Inglés, and Hidalgo (1999), and they found good psychometric properties.

*Public speaking self-efficacy questionnaire (PSSEQ) (adapted from Bados, 1986). This instrument has been adapted from the questionnaire "self-efficacy for speaking in public" (Bados, 1986). This measure was validated in Spanish populations and showed good psychometric properties. It assesses the degree of self-efficacy for speaking in public on a $0-10$ scale ( 0 being "I can't do that at all," and 10 being "I can definitely do that").

*Target behaviors (adapted from Marks \& Mathews, 1979). The therapist and the patient established two target behaviors. She had to rate the level of fear and avoidance she had in several social situations on a scale of $0-10$ ( 0 being "no fear at all; I never avoid," and 10 being "severe fear; I always avoid"). Each social situation is a different scenario of the program. 
Subjective units of discomfort (adapted from wolpe, 1969). During the exposure sessions the patient reports the level of fear regarding confronting the feared situation on a scale from 0 to 10 .

*Impairment questionnaire (adapted from echeburúa, Corral, \& Fernández-Montalvo, 2000). This measure was adapted from Echeburúa and colleagues (2000). This questionnaire measures the level of impairment of the problem caused in different areas of the patient's life, although we centered our attention on work impairment. A five-point Likert scale from 0 ("no impairment") to 4 ("great impairment") was used.

\section{Attitudes Toward the Treatment Programs Measures}

Confidence in the internet and computer expertise (CICE) (Botella et al., 2007). We created a four-item questionnaire to assess confidence in the following: Internet as a medium for finding information to solve personal problems, computers as an instrument that can help solve psychological problems, treatment program, and ability to selfapply this treatment. The items were rated on a scale of 0 (total disagreement) to 10 (total agreement). The maximum score on this measure was 40 .

Satisfaction with treatment (adapted from Borkovec \& Nau, 1972). Our research group developed a questionnaire to assess satisfaction with the treatment. The content of the items covered how logical the treatment is, to what extend it satisfies the patient, if it is useful to treat other problems, its usefulness for the patient's problem, and to what extent it could be aversive. An 11-point Likert scale from 0 to 10 was used.

\section{Apparatus and Software}

The hardware used was a Pentium II personal computer $(400 \mathrm{~Hz}$, $256 \mathrm{MB}$ of RAM, and graphics card of $64 \mathrm{MB}$ ). The peripherals the patient used were a monitor, a mouse, a keyboard, and speakers. A modem was used for the Internet access. The software used to develop the treatment was Microsoft Windows 98, Windows Media Player, and Internet Explorer 5 or 6 . An important technical aspect of the Talk to Me is video streaming. This technology makes it possible to watch and hear videos in real-time through the Internet. 


\section{Procedure}

After a first screening, two diagnostic sessions were conducted in which the diagnosis of social phobia was established by one experienced psychologist and confirmed by an independent assessor. After that the patient was asked if she agreed to use an Internet-based selfhelp program for the treatment of her problem. She was very pleased to use Talk to Me and signed a consent form. At pretest the patient completed the self-report measures previously described. After that the psychologist explained in detail to the patient how to use the Talk to Me program. The patient self-applied the program at home without any difficulty. She finished the program in two months. During this time she received four education sessions, eight exposure sessions, and one prevention relapse session. After the treatment and at one year follow-up she completed the same measures.

\section{Treatment}

Talk to Me is an Internet-based self-help program created to guide the patient through the whole therapeutic process. This program is composed of an assessment protocol and a structured treatment protocol. Both components are comprised of separate blocks through which users must progress in a specified order, which ensures that the patient does not skip any steps in the treatment. Furthermore, Talk to Me assesses the patient periodically during the treatment and makes subsequent treatment recommendations.

Initially the system provides the patient information about the program to ensure ethical issues are addressed: information about the research and the clinical team that developed the program, description of the problem that the system addresses (fear of public speaking), rationale about the treatment, safety measures to ensure confidentiality, and finally how to use the system.

Assessment. The assessment protocol presents a number of self-report instruments for evaluating the problem. The program uses the outcomes of these measures to establish the patient diagnosis and to obtain detailed information about the problem. Talk to $\mathrm{Me}$ creates an individualized treatment for each user based on pretreatment assessment. Depending on the initial scores, the program informs the patient that he or she can benefit from the program or 
it recommends looking for another kind of mental health help. This decision is made taking into account the criteria established by the standardized self-reports included in the assessment protocol. The next step is to construct a hierarchy of targeted behaviors for the exposure task. As previously stated, in the measures section the key assessment instruments of Talk to Me are marked with an asterisk.

Scenarios. This program offers six scenarios consisting of videos of real videotaped audiences that simulate different public speaking situations. Every patient confronts some of the scenarios depending on the pretreatment assessment. The reader can see a sample of each scenario at the following website: www.internetmeayuda.com/ inicio_en.htm.

- The class: The program has two class scenarios; one is comprised of 7 to 8 students and the other of 20 to 30 students. The public is seated, and the students are looking at the user situated in front of the class.

- The conference: The scenario depicts a formal situation; the user must give a talk at a conference. The audience is composed of 50 people wearing formal clothes, who are gazing toward the user.

- The oral exam: There are different scenarios depending on the number of professors (2, 3, or 5) and the gender (all women, all men, and mixed tribunals). The user must perform an oral exam.

- The work meeting: The patient must present a project in front of 9 colleagues and the boss. All of them are seated around a conference table.

- Job interview: There are two scenarios, in one of them the interviewer is a woman and in the other a man. The interviewer asks some questions of the patient.

- The wedding: The patient and other people are seated around a restaurant table, and they talk to one another.

The treatment. After the assessment, the treatment starts. It is a cognitive-behavioral treatment for fear of public speaking comprised of three components: education, cognitive-therapy, and exposure. Exposure and cognitive therapy are included in the list of empirically 
supported treatments (Task Force of Promotion and Dissemination of Psychological procedures, 1995, last updated Woody \& Sanderson, 1998).

The educational component of the program gives information about the rationale of the treatment and its components. After presenting this information, Talk to Me asks some questions that the user must answer correctly to progress to a new step. If the user answers incorrectly, he or she has to read the information again. The cognitive therapy component involves teaching users to identify and challenge automatic thoughts. The program teaches the patient to develop rational responses in public speaking situations by using questions. During the exposure the user is instructed to use the learned skills.

A very important treatment component is exposure. Talk to Me puts into order several scenarios depending on the scores obtained in the assessment phase regarding the target behaviors. Therefore, it presents a hierarchy of scenarios that the user must confront. Each scenario has several modulators to make the hierarchy: number of people, gender, difficulty of speech, and level of audience attention. The system selects the scenarios that the user fears more than 4 in a 0 to 10 scale.

At the beginnig of the exposure sessions Talk to Me instructs the user via text about what he or she has to do. Before confronting the scenario the program asks the degree of fear, avoidance, and belief on an automatic thought. Next the program has a narrative to introduce the user to the situation. This narrative explains the situation that the patient is going to confront. The next step is to give a speech in front of a virtual audience. The system asks the user's degree of fear every five minutes. In the case that the patient wants to give up the exposure before a fear decrease Talk to Me advises him or her to continue in the situation. The length of the exposure is not limited because there are individual differences regarding habituation. After the exposure session the program presents the user with graphs about the progress of anxiety during the task. Furthermore, Talk to Me reinforces the patient for the effort and success achieved. Each exposure session follows the same procedure. The treatment concludes when the user has overcome all of the target behaviors.

Another relevant point is that the program does not give real-world self-exposure instructions because our purpose is to find out the telepsychology program efficacy. An exposure program 
usually includes self-exposure instructions in order to carry out in vivo exposure between sessions. In this case we did not ask the patient to carry out in vivo self-exposure tasks because then it would have been very difficult to distinguish the contribution of the self-applied telepsychology program from the contribution of the in vivo exposure tasks in the treatment success.

\section{RESULTS}

\section{Progress Within Sessions}

Before and after every video exposure session, the program asks the degree of fear and avoidance related to the social situation that the patient was going to confront. Figure 1 shows the evolution of those measures along the eight exposure sessions. After each exposure session a reduction in fear was observed. Furthermore, Talk to Me asked the degree of fear (SUDs) every five minutes during each exposure session. We can see in Figure 2 the degree of fear during the first and the last exposure session. We observe a decrease of fear along both sessions.

\section{Other Efficacy Measures}

After the treatment, as can be seen in Table 1, there was an important reduction in the measures related to social phobia. This reduction was so pronounced in specific measures of fear of public speaking that patient's scores were similar to the scores of normal samples. This improvement was maintained at one-year follow-up.

\section{Attitudes Toward the Internet-Based Treatment Program}

The patient accepted the program before and after the treatment in the same rate. She reported high motivation toward Talk to Me before starting this program. Furthermore, the patient reported high confidence in the Internet to find information to solve personal problems, she trusted computers to solve her problem and she trusted the treatment and herself to self-apply Talk to Me. As we can see in Table 2 the confidence results were maintained at posttest and at one-year follow-up.

At the end of the treatment the patient thought that the program was logical, useful for her problem, and useful for other problems. 
FIGURE 1. Ratings Regarding the Target Behaviors Taken in Session Before and After Each Exposure Session Note: Degree of fear and avoidance measured by the fear and avoidance scale adapted from Marks \& Mathews (1979). We also included the maximum degree of fear during the session. The diamond line represents the ratings before exposure at the beginning of the session, the triangle represents the ratings during the exposure session, and the asterick line represents the ratings after the exposure task at the end of the session.

FEAR

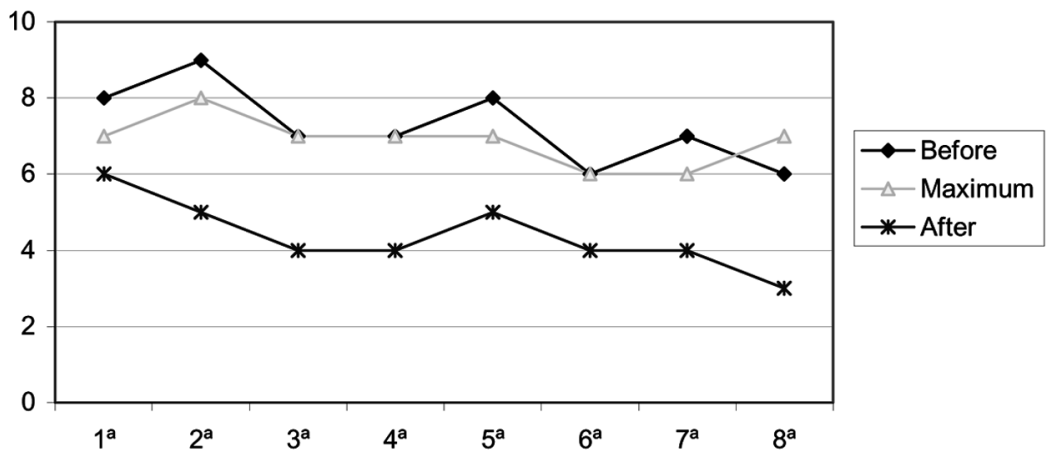

AVOIDANCE

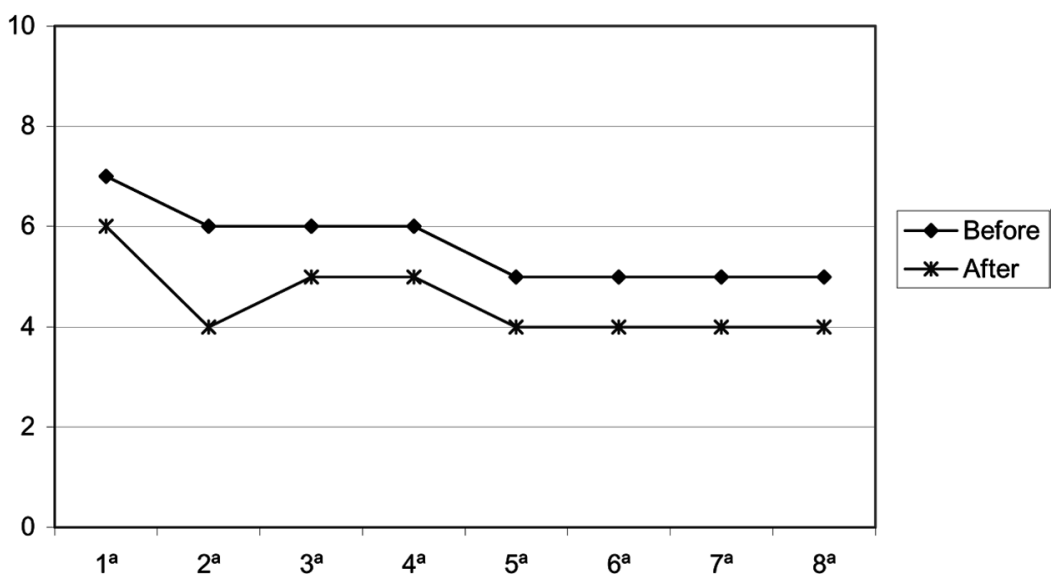

She also was satisfied with the treatment and would recommend the program to others with the same problem. With regard to components and scenarios, the patient found them very useful. 
FIGURE 2. Degree of Fear (SUDs from 0 to 10) During the First Session (Exposure to a Class) and the Last Session (Oral Exam)
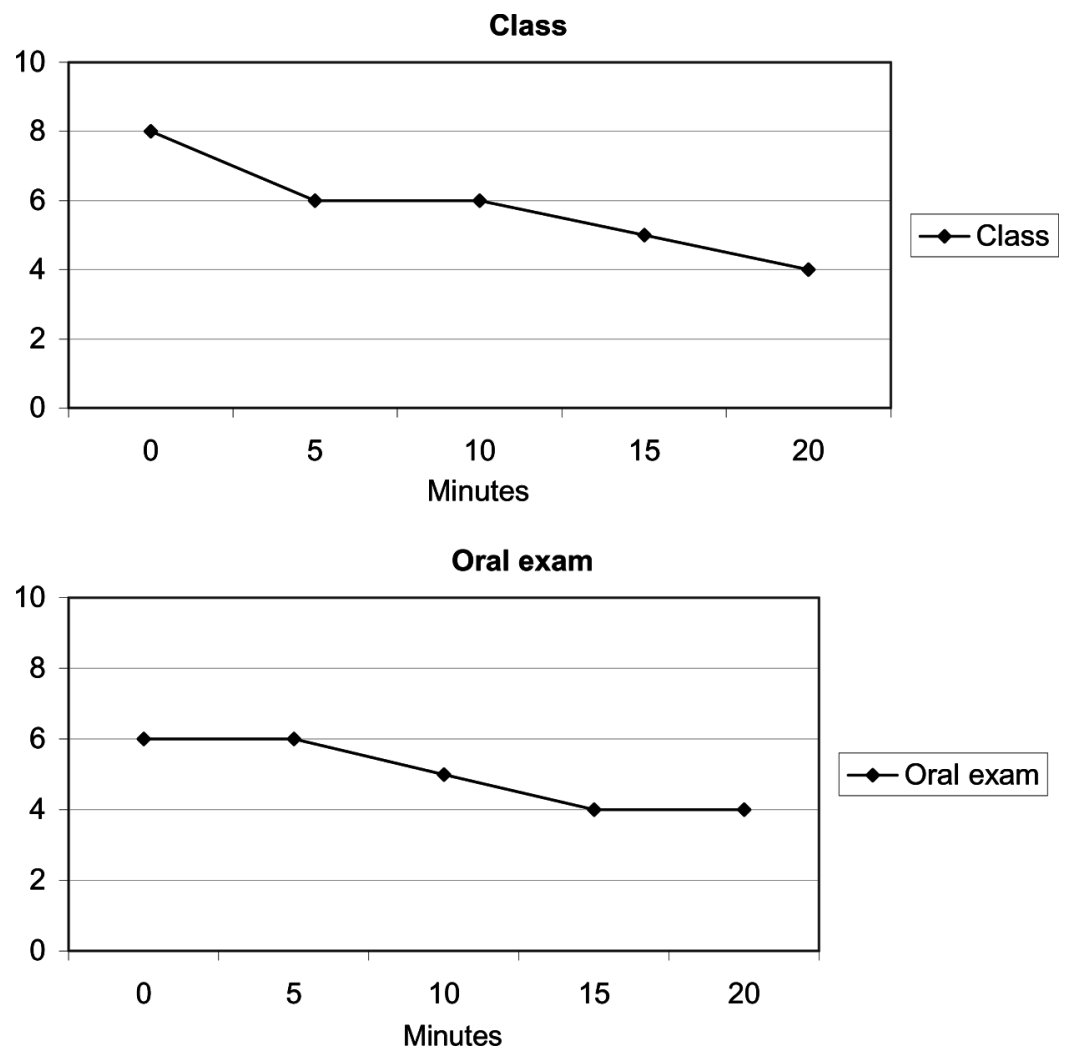

\section{DISCUSSION}

This work provides preliminary data about the utility of an Internetbased self-help program for the fear of public speaking and presents data on the degree of fear within exposure sessions. We saw a reduction in the clinical measures of social phobia from pretest to posttest, and this improvement was maintained at one-year follow-up. Although not the only component, video exposure was the main component of the program. As we see in Figure 2, the degree of fear decreased across the exposure session. Furthermore, the fear and avoidance degree related to the target behavior was reduced from the beginning to the end of each session and across the sessions (see Figure 1). With regard 
TABLE 1. Measures of Efficacy at Pretest, Posttest and One-Year Follow-up

\begin{tabular}{llrrrr}
\hline Target Behaviors & & Pre & Post & FU & \\
\hline To present a project in class/“People & Fear & 6 & 2 & 2 & Clinical sample \\
$\quad$ will think I'm stupid.” & Avoidance & 10 & 0 & 0 & \\
To give a talk/“People will think & Fear & 6 & 3 & 3 & Gallego (2006) \\
$\quad$ I'm stupid." & Avoidance & 10 & 0 & 0 & \\
\hline Social Phobia Measures & & & & Mean (SD) \\
\hline BFNE & 32 & 22 & 20 & $43.03(9.15)$ \\
SSPS-P & 14 & 21 & 22 & $12.38(5.78)$ \\
SSPS-N & 4 & 0 & 0 & $9.90(6.68)$ \\
PRCS & 121 & 79 & 99 & $135.25(19.48)$ \\
PSSEQ & 29 & 56 & 50 & $23.86(11.56)$ \\
Severity & 3 & 1 & 1 & $2.60(.85)$ \\
Impairment & & & & \\
Work impairment & 2 & 0 & 0 & $2.82(1.30)$ \\
\hline
\end{tabular}

Note: FU: one year follow-up; BFNE: brief version of the fear of negative evaluation scale; SSPS-P: self-statements during public speaking-positive subscale; SSPS-N: self-statements during public speaking-negative subscale; PRCS: personal report of confidence as a speaker; PSSEQ: public speaking self-efficacy questionnaire.

TABLE 2. Variables of the Talk to Me Acceptance

\begin{tabular}{lccc}
\hline & Pretest & Posttest & Follow-up \\
\hline Measure & 10 & & \\
Motivation & 9 & 6 & 9 \\
Internet solves problems & 9 & 10 & 9 \\
Trust computers & 10 & 10 & 10 \\
Trust treatment & 10 & 10 & 10 \\
Trust myself & & 10 & 10 \\
Logic & & 10 & 10 \\
Satisfaction & & 10 & 10 \\
Recommendation to others & & 10 & 10 \\
Utility for other problems & & 10 & 10 \\
Utility for the patient & & 0 & 0 \\
Aversiveness & & 10 & 10 \\
Components utility & 10 & 10 \\
Education & & 10 & 8 \\
Cognitive & & & \\
Exposure & 10 & 9 \\
Scenarios utility & 10 & 10 \\
Class & 10 & \\
Oral exam & & & \\
Conference & & & \\
\hline
\end{tabular}


to the results in the fear of public speaking measures at pretest, the patient's scores could be considered as belonging to a clinical population. After the treatment, the reduction of the scores was so dramatic that they were similar to a normal sample (see Table 1). The patient found the treatment program useful in overcoming her problem. In addition, she trusted Talk to Me and was satified.

We would like to highlight a relevant result: the maintenance of the therapeutic gains at one-year follow-up. The patient did not receive any other psychological or pharmacological treatment for social phobia during the follow-up period. In general, she maintained the goals achieved, and in some measures such as the PSSEQ (a self-efficacy questionnaire) she even improved, although in others such as the PRCS (personal report of confidence as a public speaker) she did not. In the last follow-up assessment we saw that the follow-up score was lower than the pretest score. Internet-based self-help efficacy is a recent line of research, and although there are some empirical reports of the efficacy of these treatments, none of them used exposure to videos for the treatment of social phobia. Our work presents preliminary data about the degree of fear within exposure session. These data showed that Talk to Me was able to activate fear and promote habituation, key elements for the success of exposure.

Another important point is that changes observed in relevant measures were clinically significant. On the one hand, the patient achieved the therapeutic goals established at pretest. On the other hand, her scores in fear of public speaking measures were similar to the scores of a clinical sample at pretest and they were reduced at posttest.

Furthermore, the patient reported at follow-up that she had presented a project in class without too much anxiety and she performed it very well, in fact the vote she got in the project was very good. This is a crucial issue in etherapy. The patient habituated to videotaped audiences and the outcomes generalized to real public speaking situations. This is a promising result, but this is only a case study and further research is needed in this particular matter.

The acceptance of this treatment by the patient is crucial for its administration. Before the treatment she was very motivated to begin the program and she had confidence in Talk to Me. After the treatment she also trusted the program, probably because this program met patient expectations. In addition, she thought that the treatment 
was logical and useful for her and for other problems. Regarding the components utility, she reported high utility for all of them (education, cognitive, and exposure) and for the three scenarios that she had confronted.

We can conclude that this study offers preliminary data about the efficacy and effectiveness (or clinical utility) of an Internet-based selfhelp program. Also, we have seen the importance of the video exposure component. Internet-based treatments can help patients overcome some limitations of the cognitive-behavioral treatments for this disorder.

This study is not free of limitations. We have to take into account that it is a case study and the data are preliminary, so we cannot draw strong conclusions. Because of this we have conducted a controlled study that compare Talk to Me with the same treatment administered by a therapist and a waiting-list control group (Gallego, 2006). Talk to Me was equally effective as a therapist-administered treatment and more effective than a waiting-list condition.

Talk to Me involves an intensive assessment throughout all of the treatment process. This could be seen as a limitation because we are asking more effort of the patient. On the other hand, we think the assessment during the treatment is important because it is selfadministered. The patient has not the expertise to determine if he or she can go a step forward in the difficulty of the exposure tasks. Because of this we set the criteria using expert clinical judgment and used the system to make the decisions. Decision making based on the assessment during the treatment is needed for optimal levels of exposure to the feared situation.

Internet-based self-help programs offer some advantatges. Patients need less therapist time because they can self-administer the program at home. Another issue related to time is that they can enter the program at any time during the week, on holidays, and day or night. Confidentiality is also important. Receiving treatment at home assures a high degree of privacy. Some of these points and not having therapist contact reduces treatment costs. Regarding exposure tasks, Talk to Me offers a safe environment where the patient can explore the problem and practice the therapeutic tools.

Our work offers preliminary data that support the use of online therapy completely delivered by the user through an Internet-based program. The program was a useful and effective alternative to conducting exposure therapy in the treatment of anxiety disorders. We 
understand, however, that any case study involves considerable risk in drawing conclusions about the causes of the outcome. The patient may have been particularly motivated or may be different from others with social phobia in innumerable ways. The novelty of the program and the extensive evaluation may also have influenced the results. Further research with larger samples and controlled studies are needed.

\section{ACKNOWLEDGEMENT}

The research presented in this paper was funded in part by Ministerio de Educación y Ciencia, Spain. PROYECTOS CONSOLIDER-C (SEJ2006-14301/PSIC). CIBER Fisiopatologia de la Obesidad y Nutricion is an initiative of ISCIII.

\section{REFERENCES}

Al-Kubaiy, T., Marks, I. M., Logsdail, S., Marks, M. P., Lovell, K., Sungur, M. et al. (1992). Role of exposure home work in phobia reduction a controlled study. Behavior Therapy, 23, 599-621.

American Psychiatric Association (APA) (2000). Diagnostic and statistical manual of mental disorder (5th ed.) Washington, DC: APA (Barcelona, Masson, 2000).

Andersson, G., Carlbring, P., Holmström, A., Spartan, L., Furmark, T., NilssonIhrfelt, E., et al. (2006). Internet-based self-help with therapist feedback and in-vivo group exposure for social phobia: A behaviour controlled trial. Journal of Consulting and Clinical Psychology, 74, 677-686.

Bados, A. (1986). Análisis de componentes de un tratamiento cognitivo-somáticoconductual del miedo a hablar en público. Doctoral Dissertation, Universidad de Barcelona.

Borkovec, T. D., \& Nau, S. D. (1972). Credibility of analogue therapy rationales. Journal of Behaviour Therapy and Experimental Psychiatry, 3, 257-260.

Botella, C., Baños, R. M., Guillén, V., Perpiña, C., Alcañiz, M., \& Pons, A. (2000). Telepsychology: Public speaking fear treatment on the Internet. CyberPsychology \& Behavior, 3, 959-968.

Botella, C., Guillen, V., Baños, R. M., Garcia-Palacios, A., Gallego, M. J. \& Alcañiz, M. (2007). Telepsychology and self-help: The treatment of fear of public speaking. Cognitive and Behavioral Practice, 14, 46-57.

Botella, C., Hofmann, S. G., \& Moscovitz, D. A. (2004). A self-applied Internetbased intervention for fear of public speaking. Journal of Clinical Psychology, $60,1-10$. 
Boyd, J. H., Rae, D. S., Thompson, J.W., Burns, B. J., Bourdon, K., Locke, B. Z., et al. (1990). Phobia: Prevalence and risk factors. Social Psychiatry and Psychiatric Epidemiology, 25, 314-323.

Brown, F. W. (1998). Rural telepsychiatry. Psychiatric Services, 49, 963-964.

Butler, C., Cullington, A., Munby, M., Amies, P., \& Gelder, M. (1984). Exposure and anxiety management in the treatment of social phobia. Journal of Consulting and Clinical Psychology, 52, 642-650.

Carlbring, P., Furmark, T., Steczkó, J., Ekselius, L., \& Andersson, G. (2006). An open study of Internet-based bibliotherapy with minimal therapist contact via e-mail for social phobia. Clinical Psychology, 10, 30-38.

Carlbring, P., Gunnarsdóttir, M., Hedensjö, L., Andersson, G., Ekselius, L., \& Furmark, T. (2007). Treatment of social phobia: Randomized trial of Internetdelivered cognitive-behavioural therapy with telephone support. The British Journal of Psychiatry, 190, 123-128.

Cho, Y., \& Won, H. (1997). Cognitive assessment of social anxiety: a study on the development and validation of the social interaction self-efficacy scale. Issues in Psychological Research, 4, 397-434.

Craske, M. G., Antony, M. M., \& Barlow, D. H. (1997). Mastery of your specific phobia, therapist guide. San Antonio, TX: The Psychological Corporation.

Di Nardo, P. A., Brown, E. L., \& Barlow, D. H. (1994). Anxiety disorders interview schedule for DSM-IV: Lifetime version (ADIS-IV-L). Albany, NY: Gaywind Publications Incorporated.

Echeburúa, E., Corral, P., \& Fernández-Montalvo, J. (2000). Escala de inadaptación: Propiedades psicométricas en contextos clínicos. Análisis en contextos clínicos. Análisis y modificación de conducta, 26, 325-338.

Foa, E. B., \& Kozak, M. J. (1986). Emotional processing of fear: Exposure to corrective information. Psychological Bulletin, 99, 20-35.

Gallego, M. J. (2006). An Internet-delivered program for the treatment of fear of public speaking. Doctoral Dissertation, Universitat Jaume I.

Gallego, M. J., Botella, C., Quero, S., Baños, R. M., \& Garcia-Palacios, A. (2007). Psychometric properties of the brief version of the Fear of Negative Evaluation Scale (BFNE) in a clinical sample. Revista de Psicopatología y Psicología Clínico, $12,163-176$.

Garcia-Palacios, A., Botella, C., Hoffman, H., \& Fabregat, S. (2007, October). Comparing acceptance and refusal rates of Virtual Reality exposure vs. in vivo exposure by patients with specific phobias. Cyberpsychology and Behavior, 10(5), $722-724$.

Ghosh, A., \& Marks, I. M. (1987). Self-treatment of agoraphobia by exposure. Behavior Therapy, 18, 3-16.

Gilkinson, H. (1942). Social fears as reported by students in collage speech classes. Speech Monoghraphy, 9, 141-160.

Glasgow, R., \& Rosen, G. (1978). Behavioral bibliotherapy: A review of self-help behavior therapy manuals. Psychological Bulletin, 85, 1-23.

Guillen, V. (2001). Miedo a hablar en público: Un tratamiento autoaplicado en internet. Tesis de licenciatura no publicada, Universidad de Valencia. 
Heimberg, R. G. (1991). A manual for conducting cognitive-behavioral group therapy for social phobia. Unpublished manuscript, State University of New York at Albany, Center for Stress and Anxiety Disorders, Albany, NY.

Heimberg, R. G., Dodge, C. S., Hope, D. A., Kennedy, C. R., Zollo, L., \& Becker, R. E. (1990). Cognitive behavioral group treatment of social phobia: Comparison to a credible placebo control. Cognitive Therapy and Research, 14, 1-23.

Heimberg, R. G., Holt, C. S., Schneier, F. R., Spitzer, R. L., \& Liebowitz, M. R. (1993). The issue of subtypes in the diagnosis of social phobia. Journal of Anxiety Disorders, 7, 249-269.

Hellström, K., \& Öst, L. G. (1995). One-session therapist directed exposure vs. two forms of manual directed self-exposure in the treatment of spider phobia. Behavior Research and Therapy, 33, 959-965.

Hofmann, S. G., \& DiBartolo, P. M. (2000). An instrument to assess self-statements during public speaking: Scale development and preliminary psychometric properties. Behavior Therapy, 31, 499-515.

Kessler, R. C., McGonagle, K. A., Zhao, S., Nelson, C. B., Hughes, M., Eshleman, S., et al. (1994). Lifetime and 12-month prevalence of DSM-III-R psychiatric disorders in the United States. Archives of General Psychiatry, 51, 8-19.

Leary, M. R. (1983). A brief version of the fear of negative evaluation scale. Personality and Social Psychology Bulletin, 9, 371-375.

Magee, W. J., Eaton, W. W., Wittchen, H.U., McGonagle, K. A., \& Kessler, R. C. (1996). Agoraphobia, simple phobia, and social phobia in the National Comorbidity Survey. Archives of General Psychiatry, 53, 159-168.

Marks, I. M. (1978a). Behavioral psychotherapy of adult neurosis. In S. L. Gardfield \& a. E. Bergin (Eds.), Handbook of psychotherapy and behavior change (2nd ed.). New York: Wiley.

Marks, I. M. (1978b). Living with fear. New York: McGraw Hill.

Marks, I. M. (1992). Tratamiento de exposición en la agorafobia y el pánico. In Echeburua, E. (Ed.), Avances en el tratamiento psicológico de los trastornos de ansiedad. Madrid: Piramide.

Marks, I. M. (1987). Fear, phobias, and rituals: Panic, anxiety, and their disorders. New York: Oxford University Press.

Marks, I. M. \& Matthews, A. M. (1979). Brief standard self-rating for phobic patients. Behaviour Research and Therapy, 17, 263-267.

Mathews, A. M., Gelder, G., \& Johnston, D. W. (1981). Agoraphobia: Nature and Treatment. New Cork: Guilford.

Mattick, R. P., Peters, L., \& Clarke, J. (1989). Exposure and cognitive restructuring for social phobia: A controlled study. Behavior Therapy, 20, 3-23.

Méndez, F. J., Inglés, C. J., \& Hidalgo, M. D. (1999). Propiedades psicométricas del cuestionario de confianza para hablar en público: estudio con una muestra de alumnos de enseñanzas medias. Psicothema, 1, 65-74.

Park, J. M., Mataix-Cols, D., Marks, I. M., Ngamthipwatthna, T., Marks, M., Araya, R. et al. (2001). Two-year follow-up after a randomized controlled trial of self- and clinician-accompanied exposure for phobia/panic disorders. The British Journal of Psychiatry: The Journal of Mental Science, 178, 543-548. 
Paul, G. L. (1966). Insight vs. desensitization in psychotherapy. Standford, CA: Standford University Press.

Pollard, C. A., \& Henderson, J. F. (1988). Four types of social phobia in a community sample. Journal of Nervous and Mental Disease, 176, 440-445.

Roy, S., Kingler, E., Légeron, P., Lauer, F., Chemin, I. \& Nugues, P. (2003). Definition of a VR-based protocol to treat social phobia. CyberPsychology \& Behavior, 6, 411-420.

Schneider, A. J., Mataix-Cols, D., Marks, I. M., \& Bachofen, M. (2005). Internetguided self-help with or without exposure therapy for phobic and panic disorders. Psychotherapy and Psychosomatics, 74, 154-164.

Stein, M. B., Walter, J. R., \& Forde, D. R., (1996). Public speaking fears in a community sample: prevalence, impact on functioning, and diagnostic classification. Archives of General Psychiatry, 53, 169-174.

Turner, S. M., Beidel, D. C., \& Cooley, M. (1997). Social effectiveness therapy: A program for overcoming social anxiety and phobia. Toronto: Multi-Health Systems.

Turner, S. M., Beidel, D. C., \& Jacob, R. G. (1994). Social phobia: A comparison of behavior therapy and atenolol. Journal of Consulting and Clinical Psychology, 62, 350-358.

Üst, L. G., Salkovskis, P. M., \& Hellström, K. (1991). One-session therapist directed exposure vs. self-exposure in the treatment of spider phobia. Behavior Therapy, 22, 407-422.

Wolpe, J. (1969). The practice of behavior therapy. New York: Pergamon Press.

Woody, S. R., \& Sanderson, W. C. (1998). Manuals for empirically supported treatments: 1998 update. The Clinical Psychologist, 51, 17-21.

Received: $08 / 09 / 07$

Revised: $10 / 02 / 07$

Accepted: 01/07/08 